sional opinions of technical officers too frequently are not given the due weight which they deserve. Science has done much for the Civil Service; it has not, in return, received the recognition which it merits.

The question arises: How can the defects and abuses known to exist in the Civil Service be best corrected? Remedies there are, some of which have been made public. Such remedies are not competitive inter se : they can be applied concurrently, and are capable both of promoting the welfare of the Civil Service and at the same time of adequately protecting the public interest. The report of Sir J. J. Thomson's Committee on the position of natural science in the educational system of Great Britain (Cd. goII) contains two important recommendations having these objects in view, viz. (a) that all candidates for the competitive examination for the Home and Indian Civil Services should supply evidence of a continuous training in science extending over several years; and $(b)$ that many posts in the public services should be filled by men selected, not by the ordinary competitive examination, but, at a riper age, on the ground of high scientific qualifications and professional experience.

In view of the present organisation of the Civil Service, it is very certain that the adoption of the first of the foregoing recommendations alone, as a solitary and isolated measure of reform, will not cure the defects known to exist in the Service. The scheme will do little to provide the Civil Service with a sufficient number of men of high scientific attainments and proficient technical knowledge capable of administering the affairs of a modern State in the spirit of progressive knowledge; it will not remove the schism between the administrative and technical staffs of the same Department, a schism which, unfortunately, exists in some Departments to-day.

As regards the second of the above recommendations, presumably the intention is that the men of riper years selected on the ground of professional experience shall fill some of the high administrative posts in the higher division. If this is so, the recommendation is an admirable one and. worthy of immediate adoption. But the question arises whether the State will provide remuneration at a rate high enough to secure for the public services men of sufficiently good abilities. Unless it does so, nothing will be gained by the adoption of the recommendation.

A Government pension under the special provisions contained in section iv. of the Superannuation Act, 1859 (22 Vict., c. 26)-i.e. one calculated at a higher rate than the ordinary scalemight possibly, in some instances, have been considered by candidates a sufficient compensation for the lower rates of salary prevailing in the Civil Service as compared with those paid by private employers and public corporations. Bitt this feature of the Government pension scheme, provided originally to meet the cases of the kind now under consideration, has seased to exist: the section of the Act of 1859 in question was reNO. 2545 , VOL. IOI] pealed by section $v$. of the Superannuation Act, I9I4 (4 \& 5 Geo. V., c. 86).

The only effective remedy for curing the ills from which the Civil Service is suffering at the present time consists in a root-and-branch reform, a reform involving the re-fashioning of its entire framework and fabric. No dangerous or expensive experiments are necessary for the purpose of "trying out" and "proving in" a new organisation: a model well worthy of imitation exists in the Swedish Civil: Service, with its administrative boards. This model could easily be adapted to meet the requirements of this country; the system of administrative boards would provide a means for utilising to the best advantage the existing administrative and technical officers in the Civil Service by associating with them men of large business and professional experience drawn from outside the public services. A reorganisation on the lines here suggested would naturally bring in its train the recognition of the necessity for a more widespread knowledge of science in the Civil Service. Simultaneously, effect could be given to the recommendation regarding the appointment, to permanent posts of the higher division, of men of professional experience as recommended by Sir J. J. Thomson's Committee. Finally, with the advent of the administrative boards would disappear the methods of administration based on the despot's maxim, Divide et impera, methods which continue to have a vogue in certain Departments. Such methods, it is scarcely necessary to point out, are extremely wasteful, and can have no place in any régime which relies for its prosperity and efficiency on science.

\section{THE EARLY HISTORY OF THE SOLAR SYSTEM.}

A COMMON feature of the older theories of the origin of the solar system is that they all suppose it to have been derived from a more or less symmetrical rotating nebula in a gaseous or quasi-gaseous state. By some process, the details of which differ in different theories, this mass is supposed to have condensed locally to form the sun and planets. A recent paper by Jeans has indicated a way of examining whether such condensation is possible. Viscosity is insufficient to cause a mass so large as the primitive nebula to rotate like a rigid body; each part would revolve practically independently around the centre under gravity, and the matter near any point, on account of the differences between the velocities of different parts, would be in a state of rotation with an angular velocity different from that of its revolution as a whole. It is, however, easily shown that the two are of the same order of magnitude. Now a mass cannot condense locally unless the density is so great that mutual gravitation is enough to balance the centrifugal force due to the rotation, and this indicates that, before condensation started at distance $r$ from the centre, the density there must have been at least com- 
parable with the mean density of a sphere of radius $r$ and mass equal to that of the sun. Planets having been formed at many different distances from the sun, it follows that the mass must have originally been widely distributed through the system. The distribution of density and velocity being thus known within sufficiently narrow limits, it can be shown by the principle of the constancy of angular momentum that if planets of the sizes of ours were formed, the resulting central body could not possibly rotate so slowly as the actual sun. There is no agency capable of reducing this rotation, and it seems necessary to abandon completely those hypotheses that require the solar system to have been formed by the gradual condensation of a nebula.

We are led to inquire next whether planets could come into being by a more rapid or catastrophic process. Projection from the sun is not a possible origin, for a body started in this way must necessarily strike the sun again on its return and be reabsorbed; further, there is no reason why all should revolve in the same direction. The tidal theory appears to give a better account of the present state of the system. According to this, a star much more massive than the sun approached it very closely, and raised on opposite sides of it two projections, just as the moon raises tides in the earth; but the scale of the disturbance was in this case so enormous that the sun's gravitation was unable to prevent a rupture from occurring. "Thus either one or two streams of matter were shot out in a time comparable with a few months or years. Being longitudinally unstable, they broke up into a series of detached masses, perhaps before the parts projected later had actually separated from the sun. That such rupture could occur has been proved by Jeans. The attraction of the disturbing body produced the direct revolution (in the same sense as the motion of the star relative to the sun); some of the revolving matter returned into the sun and gave it a direct rotation. The angular momentum thus acquired was, of course, derived from the transverse motion of the disturbing body relative tc the sun.

The size of the deformed body has little influence on its chance of being broken up. Thus the detached masses might well have produced systems of satellites and developed direct rotations in the majority of cases, though complete uniformity could scarcely be expeeted on account of the number of complicating factors. The fission would cease when the star had receded a sufficient distance; thus the outer nuclei, being the first ejected, would produce most satellites. It seems possible also that some of these would be formed when the nucleus and the sun were on opposite sides of the star, and that the motion would therefore be retrograde. All the bodies, having recently formed part of the sun, would naturally be very hot.

The system after the passage of the star would therefore include a central sun surrounded by a number of heated planets, moving in direct orbits, and attended by satellites; the most reNO. 2545 , VOL. IOI] mote planets would have most satellites. The rotation of the sun would be direct; the rotation of each planet would be in the same sense as the revolution of most of its satellites, and in most cases this also would be direct, though a few exceptions might well occur, especially in the outermost sub-systems. In every point this agrees with the existing solar system. The heated interior of the earth, the building of mountains by compression, and the present heated state of the greater planets are readily accounted for. The occurrence of three retrograde satellites on the outskirts of otherwise direct sub-systems presents a difficulty, but not, I think, a serious one.

In addition to the planets and satellites, however, there would be a considerable amount of gaseous matter too light to be condensed into the nuclei, and probably consisting mainly of hydrogen. This would be pushed round by the planets as they moved, but its resistance to oscillatory motions would steadily reduce the eccentricities of their orbits, which would initially be considerable. At the same time its own viscosity and diffusion would cause it partly to dissipate into outer space and partly to be reabsorbed into the sun. 'The zodiacal light is probably the last remnant of it. The actual eccentricities of the planetary orbits being now small, but definitely different from zero, it seems that the time the medium took to degenerate and the time needed to produce a considerable effect on the eccentricities must have been of the same order of magnitude. These are capable of being estimated in terms of the density of the medium, the first being proportional and the second inversely proportional to it. Thus the condition that they are of the same order of magnitude makes it possible to estimate very roughly both the density and the time needed for the changes, which is found to be of the order of $3 \times 10^{9}$ years, as nearly equal as could be expected to the age of the earth indicated by its radio-active constituents (about $\mathrm{r} 6 \times 10^{9}$ years). At the same time large condensations would form around the larger planets, and the resistances offered by these would be so great, being proportional to the squares of the masses, that their eccentricities would diminish rapidly. Thus it would be expected that, on the whole, the larger planets would have smaller eccentricities than the smaller ones; this is the case, Mercury and Mars having larger eccentricities than Jupiter, Saturn, and Uranus, and the earth than Neptune. Venus has a somewhat smaller eccentricity than Neptune, but otherwise the agreement is remarkable.

It is certain that on the tidal theory the primitive nuclei must have been very hot, and probably fluid. Whether the larget ones were liquid or gaseous is very doubtful, but the smaller ones, including the asteroids and most of the satellites, can be shown to possess too little gravitative power to have been able to hold together in the gaseous state, and must therefore have been liquid or solid at the start. The satellites probably liquefied almost as soon as they were expelled from their primaries or from the sun, on 
account of the reduction in temperature caused by the release of pressure; solidification would occur more slowly. The origin of the asteroids presents greater difficulties. They may have started as independent planets of exceptionally small size; but the fact that none of their mean distances is greater than that of Jupiter, and only one is less than that of Mars, indicates a closer relationship between their origins. Several possible explanations can be advanced; the writer inclines to the belief that they were formed by the close approach of a primitive planet to Jupiter, leading to tidal disruption.

The large size of the moon relative to the earth suggests a fundamental difference of origin between it and the other satellites. It seems likely (indeed, on the theory of a formerly heated earth it is almost certain) that it was once much nearer the earth than it is now, and has receded on ac count of the friction of the lunar tides. It is natural to think that just before this state of motion with a comparatively small separation between their surfaces the earth and moon formed one body The rotation would then be so rapid that the longest free period of the mass was nearly equal to the period of the semi-diurnal solar tide, which was consequently enormously magnified by resonance; and it is highly probable that the deformation became so great that the mass separated into two parts. This is not the only conceivable origin of the moon that would be consistent with the tidal theory; but several peculiarities in our sub-system suggest that it is the most likely. No other satellite in the system can have been formed in this way.

As has just been remarked, lunar tidal friction has probably been the predominant cause in the evolution of the earth and moon. No other satellite can raise tides of such importance; but those raised in Mercury by the sun must have been much more effective in reducing the rotation of this planet. Now from the fact that Mercury has no satellite we may infer that it never rotated so fast as the earth did before the moon was formed; and therefore the solar tides will have been able to reduce its rotation so as to make it always keep the same face towards the sun, which again agrees with observation.

Every satellite except the moon has probably been more influenced in its orbital motion by the resisting medium than by tidal friction. The most striking effect of the medium being to reduce the eccentricities of orbits, this accounts for the almost perfect circularity of the orbits of most satellites, especially those nearest their primaries, where the density of the medium was probably greatest. The effect of tidal friction on the eccentricities is not certain, depending on certain unknown physical quantities.

It may be said finally that at every point where the tidal hypothesis has been tested it agrees with dynamical theory and with observation. Several facts otherwise unaccounted for are explained by it, and nothing has yet been discovered to be definitely opposed to it, though a few difficulties, such as the origin of comets and meteor swarms, still remain.

\section{REFERENCES.}

J. H. Jeans: "The Part Played by Rotation in Cosmic Evolution," Monthly Notices of Royal Astronomical Society, vol. 1xxvii., I9 I7, pp. I86-99; "Note on the Action of Viscosity in Gaseous and Nebular Masses," loc. cit., pp. 200-4; "The Motion of Tidally Distorted Masses, with Special Reference to Theories of Cosmogony," Memoirs of R.A.S., vol Ixii., 1917, pp. $1-48$.

Harold Jeffreys: "The Resonance Theory of the Origin of the Moon," Monthly Notices, vol. 1xxviii., I9I7, pp. II6-3I; "On the Early History of the Solar System," loc. cit., pp. 424-4I.

Harold Jeffreys.

\section{GRASSLAND AND FOOD SUPPLIES.}

THE persistent criticism, with which the Agriculture has been assailed has been intensified of late with the evidence of failure, total or partial, of some of the crops grown this year on newly ploughed grass land. The columns of the daily Press have been freely used, and have revealed much division of opinion amongst practical agriculturists as to the measure of success or failure in different areas. A new note has been introduced into the discussion by a letter from the Duke of Marlborough in the Times of July 30 , in which he endeavours to demonstrate from the publications of the Board that the policy is fundamentally unsound.

Basing his criticism upon a leaflet issued by the Board in the spring of last year, his Grace argues that, so far from the data there given proving that an increase of food supply can be expected from the ploughing-up of grass and growing corn, they demonstrate rather that the chances of securing such an increase are very speculative and scarcely likely to be realised. The facts are not disclosed that the leaflet in question deals with the production of winter food for cows, and therefore only indirectly for the human population; and, further, that the dairy farmer is advised that a much greater return of milk-producing food can be expected from growing root crops rather than corn.

The basis of comparison of the feeding values of the crops adopted in the leaflet is quite inapplicable to the assessment of the relative returns for human feeding, since for the latter purpose grass is worthless until converted into other forms which represent only a fraction of the weight of grass harvested, whereas the corn crops, by the simple and expeditious process of milling, yield anything from 60 per cent. upwards of the weight of the grain in a form directly usable for human consumption, whilst, in addition, the accompanying straw, according to its nature, when used for food production purposes, will be roughly equal to one-half its weight of hay. The Duke of Marlborough anticipates this objection by pointing out that, so far as the oat crop, at any rate, is concerned, only a small proportion of it has, in actual fact, been directly utilised for human con-

NO. 2545 , VOL. IOI] 